\title{
RESEARCH
}

Open Access

\section{Functionally enhanced placenta-derived mesenchymal stem cells inhibit adipogenesis in orbital fibroblasts with Graves' ophthalmopathy}

\author{
Jae Yeon Kim', Sohae Park', Hyun-Jung Lee ${ }^{2}$, Helen Lew ${ }^{3}$ and Gi Jin Kim ${ }^{1 *}$ (D)
}

\begin{abstract}
Background: Placenta-derived mesenchymal stem cells (PD-MSCS) have unique immunomodulatory properties. Phosphatase of regenerating liver-1 (PRL-1) regulates the self-renewal ability of stem cells and promotes proliferation. Graves' ophthalmopathy $(\mathrm{GO})$ is an autoimmune inflammatory disease of the orbit and is characterized by increased orbital levels of adipose tissue. Here, we evaluated the therapeutic mechanism for regulation of adipogenesis by PRL-1-overexpressing PD-MSCs (PD-MSCs ${ }^{\text {PRL-1 }}$, PRL-1+) in orbital fibroblast (OF) with GO patients.

Methods: PD-MSCs isolated from human placenta were transfected with the PRL-1 gene using nonviral transfection method. Primary OFs were isolated from orbital adipose tissue specimens from GO patients. After maturation as adipogenic differentiation, normal and GO-derived OFs were cocultured with naïve and PD-MSCs ${ }^{\text {PRL-1 }}$. We analyzed the protein levels of adipogenesis markers and their signaling pathways in OFs from GO patients.

Results: The characteristics of PD-MSCs ${ }^{\text {PRL-1 }}$ were similar to those of naive cells. OFs from GO patients induced adipocyte differentiation and had significantly decreased a lipid accumulation after coculture with PD-MSCS ${ }^{\text {PRL-1 }}$ compared to naïve cells. The mRNA and protein expression of adipogenic markers was decreased in PD-MSCs ${ }^{\text {PRL-1. }}$. Insulin-like growth factorbinding proteins (IGFBPs) secreting PD-MSCs ${ }^{\text {PRL-1 }}$ downregulated the phosphorylated PI3K/AKT/mTOR expression in OFs from GO patients. Interestingly, IGFBP2, $-4,-6$, and -7 expression in PD-MSCSPRL-1, which was mediated by integrin alpha 4 (ITGA4) and beta 7 (ITGB7), was higher than that in naïve cells and upregulated phosphorylated FAK downstream factor.

Conclusion: In summary, IGFBPs secreting PD-MSC ${ }^{\text {PRL-1 }}$ inhibit adipogenesis in OFs from GO patients by upregulating phosphorylated FAK and downregulating PI3K/AKT/mTOR signaling pathway. The functional enhancement of PD-MSCS by nonviral gene modification provides a novel therapeutic strategy for the treatment of degenerative diseases.
\end{abstract}

Keywords: Adipogenesis, Graves' ophthalmopathy, Gene modification, Phosphatase of regenerating liver-1, Placentaderived mesenchymal stem cells

\footnotetext{
* Correspondence: gjkim@cha.ac.kr

'Department of Biomedical Science, CHA University, Seongnam 13488, Republic of Korea

Full list of author information is available at the end of the article
}

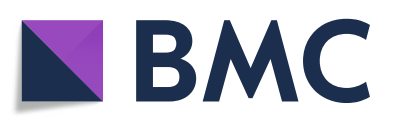

(- The Author(s). 2020 Open Access This article is licensed under a Creative Commons Attribution 4.0 International License, which permits use, sharing, adaptation, distribution and reproduction in any medium or format, as long as you give appropriate credit to the original author(s) and the source, provide a link to the Creative Commons licence, and indicate if changes were made. The images or other third party material in this article are included in the article's Creative Commons licence, unless indicated otherwise in a credit line to the material. If material is not included in the article's Creative Commons licence and your intended use is not permitted by statutory regulation or exceeds the permitted use, you will need to obtain permission directly from the copyright holder. To view a copy of this licence, visit http://creativecommons.org/licenses/by/4.0/. The Creative Commons Public Domain Dedication waiver (http://creativecommons.org/publicdomain/zero/1.0/) applies to the data made available in this article, unless otherwise stated in a credit line to the data. 


\section{Background}

Graves' ophthalmopathy (GO) is a thyroid-associated autoimmune disease of the eye that is potentially sightthreatening. The main symptoms of GO are proptosisassociated impairment of eye motility, lid retraction, de novo adipogenesis, and soft tissue inflammation. In particular, inflammatory reactions of orbital fibroblasts (OFs) are responsible for these disease symptoms [1]. Substantial evidence suggests the involvement of insulin-like growth factor 1 receptor (IGF-1R) in GO [2]. Thyroid-stimulating hormone receptor (TSHR) and IGF-1R, which are OF surface receptors, stimulate hyaluronic acid synthesis and de novo adipogenesis through peroxisome proliferatoractivated receptor gamma (PPAR- $\gamma)[3,4]$.

Based on the molecular pathogenesis of GO, medical and surgical treatments of patients with GO have been implemented. In particular, corticosteroids and orbital radiotherapy continue to be used to treat patients with GO [5]. Orbital radiotherapy combined with corticosteroids protects against disease progression by reducing compressive optic neuropathy in patients with active thyroid eye disease [6]. However, glucocorticoid therapy has a negative effect on patient hyperthyroid status and adrenal insufficiency, as well as acute liver damage, when alanine aminotransferase levels are greater than $300 \mathrm{U} / \mathrm{L}$ [7, 8]. Moreover, medical radiotherapy also resulted in the development of malignancies, depending on the age and gender of patients [9].

Placenta-derived mesenchymal stem cells (PD-MSCs) have been broadly investigated due to their multilineage differentiation potential, and these cells have especially potent immunomodulatory abilities associated with tissue repair and regenerative medicine. MSCs inhibit the proliferation of $\mathrm{T}, \mathrm{B}$, natural killer, and dendritic cells. Due to these immunoregulatory properties, the safety and clinical efficacy of MSC-based therapy has tested in preclinical [10] and transplantation studies [11]. In comparison to other MSCs, PD-MSCs have an additional immunomodulatory advantage by regulating the expression of human leukocyte antigen (HLA)-ABC and HLA-G [12]. Therefore, the therapeutic effects of PD-MSCs are considered to be associated with immunosuppressionmediated replacement of damaged tissues.

Phosphatase of regenerating liver-1 (PRL-1), also known as protein tyrosine phosphatase type IVA member 1 (PTP4A1) and PTPCAAX1, is a member of a small class of prenylated PTPs. PRL-1 was originally identified as an immediate early gene during liver regeneration [13]. PRL-1 contains the C-terminal prenylation motif for farnesylation CAAX [14]. PRL-1 promotes cellular proliferation during protein prenylation, which is a posttranslational lipid modification, by upregulating RhoA via the mevalonate metabolic pathway. The major enzyme $\beta$-hydroxy $\beta$ methylglutaryl-coenzyme A (HMG-CoA) reductase regulates AMP-activated protein kinase (AMPK) during protein prenylation through PRL-1 [15]. Moreover, PRL-1 modulates the oxidative stress response in the mammalian retina [16]. PRL-2, which is in the same class and subfamily as PRL-1, plays an important role in hematopoietic stem cell self-renewal.

Recently, we reported therapeutic effects of naive PDMSCs in mice models of GO [17]. However, it is still unknown whether functionally enhanced PD-MSCs overexpressing PRL-1 (PD-MSCs ${ }^{\text {PRL-1 }}$, PRL-1+) inhibit adipogenesis in OFs from GO patients to investigate therapeutic effects.

\section{Materials and methods}

\section{Cell culture and gene transfection}

Orbital adipose tissue specimens were obtained from patients with GO $(n=3)$ during fat decompression and from control individuals without a history of GO $(n=3)$ under consent conditions. OF preparation was approved by the Institutional Review Board of CHA Bundang Medical Center, Seongnam, Republic of Korea (IRB-2018-01-007). Orbital tissue explants were minced and treated with 0.25 $\mathrm{mg} / \mathrm{mL}$ collagenase (Sigma-Aldrich, St. Louis, MO, USA) for $1 \mathrm{~h}$ at $37^{\circ} \mathrm{C}$ in a shaking incubator. After collagenase digestion, the orbital tissues were placed in culture plates with DMEM/F12 supplemented with 20\% fetal bovine serum (FBS; Gibco, Carlsbad, CA, USA) and 1\% penicillin/streptomycin (P/S; Gibco).

Placentas were collected for research purposes by the Institutional Review Board of CHA Gangnam Medical Center, Seoul, Republic of Korea (IRB 07-18). All participants provided written informed consent prior to placenta collection. PD-MSCs were isolated as previously described [18] and cultured in $\alpha$-modified minimal essential medium $(\alpha$ MEM; HyClone Logan, UT, USA) supplemented with $10 \%$ FBS (Gibco), 1\% P/S (Gibco), $1 \mu \mathrm{g} / \mathrm{mL}$ heparin (Sigma-Aldrich), and $25 \mathrm{ng} / \mathrm{mL}$ human fibroblast growth factor-4 (hFGF-4; Peprotech, Rocky Hill, NJ, USA). The PRL-1 plasmid vector was purchased from Origene (\#RG200435; Rockville, MD, USA). To induce overexpression of the PRL-1 gene, naïve PD-MSCs (passage $=7$ ) were transfected using the AMAXA nucleofector system (Lonza, Basel, Switzerland) according to the manufacturer's instructions. After transfection for $24 \mathrm{~h}$, the cells were selected by 1.5 $\mathrm{mg} / \mathrm{mL}$ neomycin. All cells were maintained at $37^{\circ} \mathrm{C}$ in a humidified atmosphere containing $5 \% \mathrm{CO}_{2}$.

Differentiation of PD-MSCs ${ }^{\text {PRL-1 }}$ and OFs from GO patients To analyze the potential of PD-MSCs ${ }^{\text {PRL-1 }}$ to differentiate into mesodermal lineages, PD-MSCs ${ }^{\text {PRL-1 }}$ (passage $=$ 5 ) were plated at a density of $5 \times 10^{3}$ cells $/ \mathrm{cm}^{2}$ in various differentiation induction media using the StemPro adipogenesis and osteogenesis differentiation kit (Gibco) according to the manufacturer's instructions. After approximately 21 days, PD-MSCs ${ }^{\text {PRL-1 }}$ were fixed in $4 \%$ paraformaldehyde and incubated for $1 \mathrm{~h}$ with Oil Red $\mathrm{O}$ 
(Sigma-Aldrich) to stain lipids to visualize lipid vesicles and von Kossa with 5\% silver nitrate (Sigma-Aldrich) under the light to evaluate the accumulation of calcium deposits.

To induce adipogenic differentiation, normal and GOderived OFs $\left(5 \times 10^{3}\right.$ cells $\left./ \mathrm{cm}^{2}\right)$ were seeded and incubated in serum-free DMEM/F12 supplemented with $33 \mu \mathrm{M}$ biotin, $17 \mu \mathrm{M}$ pantothenic acid, $10 \mu \mathrm{g} / \mathrm{mL}$ transferrin, $0.2 \mathrm{nM}$ triiodothyronine $\left(\mathrm{T}_{3}\right), 1 \mu \mathrm{M}$ insulin (all from Sigma-Aldrich), $0.2 \mu \mathrm{M}$ carbaprostacyclin $\left(\mathrm{cPGI}_{2}\right.$; Cayman Chemical, Ann Arbor, MI, USA), $1 \mu \mathrm{M}$ dexamethasone, and $0.1 \mathrm{mM}$ isobutylmethylxanthine (IBMX; all from Sigma-Aldrich) for the first 4 days. To induce the maturation of adipocytes, the medium was supplemented except $1 \mu \mathrm{M}$ dexamethasone and $0.1 \mathrm{mM}$ IBMX (all from Sigma-Aldrich) for 6 days and was replaced every other day. Lipid accumulation and adipocyte morphology were visualized by Oil Red O staining.

\section{Coculture experiments}

To detect the inhibition of adipogenesis, normal and GO-derived OFs underwent adipogenic differentiation and were cocultured with naïve and PD-MSCs ${ }^{\mathrm{PRL}-1}(5 \times$ $10^{3}$ cells $\left./ \mathrm{cm}^{2}\right)$ in Transwell inserts $(8 \mu \mathrm{m}$ pore size; Corning, NY, USA) in $\alpha$-MEM (HyClone) supplemented with $10 \% \mathrm{FBS}$ and $1 \% \mathrm{P} / \mathrm{S}$ (all from Gibco) for $24 \mathrm{~h}$ at $37^{\circ} \mathrm{C}$ in a humidified atmosphere containing $5 \% \mathrm{CO}_{2}$.

\section{Reverse transcription polymerase chain reaction (RT-PCR) and quantitative real-time PCR (qRT-PCR)}

Total RNA was extracted using TRIzol LS reagent (Invitrogen, Carlsbad, CA, USA) according to the manufacturer's protocol. The concentration and purity of the total RNA were determined spectrophotometrically by measuring the ODs at $260 \mathrm{~nm}$ and $280 \mathrm{~nm}$. cDNA was reverse transcribed from total RNA (500 ng) by using SuperScript III reverse transcriptase (Invitrogen). To analyze stemness markers in PD-MSCs ${ }^{\text {PRL-1 }}$, PCR amplification was performed with specific primers (Table 1). $\beta$-actin was used as an internal control. The amplified PCR products were electrophoresed on $2 \%$ agarose gels containing $1.5 \mu \mathrm{g} / \mathrm{mL}$ ethidium bromide and visualized under UV light. qRT-PCR analysis was used to determine differences in gene expression. qRT-PCR was performed with primers (Table 2) and SYBR Green PCR master mix (Roche, Basel, Switzerland) in a CFX Connect $^{\text {tw }}$ Real-Time System (Bio-Rad, Hercules, CA, USA). All reactions were performed in triplicate.

\section{Flow cytometry analysis}

For immunophenotyping of cell surface antigens, thirdpassage PD-MSCs ${ }^{\text {PRL-1 }}$ were detached, stained with antibodies conjugated with fluorescein isothiocyanate (FITC) and phycoerythrin (PE) and analyzed with a FACSCalibur
Table 1 Primer sequences using reverse transcription polymerase chain reaction

\begin{tabular}{llll}
\hline Genes & & Primer sequences & Tm \\
\hline Oct4 & Forward & 5'-AGTGAGAGGCAACCTGGAGA-3' & 52 \\
& Reverse & 5'-GTGAAGTGAGGGCTCCCATA-3' & \\
Nanog & Forward & 5'-TTCTTGACTGGACCTTGTC-3' & 52 \\
& Reverse & 5'-GCTTGCCTTGCTTGAAGCA-3' & \\
Sox2 & Forward & 5'-GGGCAGCGTGTACTTATCCT-3' & 52 \\
& Reverse & 5'-AGAACCCCAAGATGCACAAC-3' & \\
HLA-G & Forward & 5'-GCGGCTACTACAACCAGAGC-3' & 58 \\
& Reverse & 5'-GCACATGGCACGTGTATCTC-3' & \\
TERT & Forward & 5'-GAGCTGACGTGGAAGATGAG-3' & 55 \\
& Reverse & 5'-CTTCAAGTGCTGTCTGATTCCAATG-3' & \\
AFP & Forward & 5'-ATGCTGCAAACTGACCACGC-3' & 55 \\
& Reverse & 5'-GCTTCGCTTGCCAATGCTT-3' & \\
Albumin & Forward & 5'-TGAGTTGCAGAAGTTCCA-3' & 60 \\
& Reverse & 5'-CCTTGGCTCAGCATAGTT-3' & \\
3-actin & Forward & 5'-TCCTTCTGCATCCTGTCAGCA-3' & 58 \\
& Reverse & 5'-CAGGAGATGGCCACTGCCGCA-3' & \\
\hline
\end{tabular}

flow cytometer (Becton Dickinson, Franklin Lakes, NJ, USA). The following monoclonal antibodies were used: CD34-PE, CD90-PE, HLA-ABC-FITC, HLA-DR-FITC (BD Bioscience, San Jose, CA, USA), CD13-PE (BioLegend, San Diego, CA, USA), CD105-FITC (R\&D Systems, Minneapolis, MN, USA), and HLA-G (Abcam, Cambridge, UK). For each sample, at least 10,000 events were acquired.

\section{Teratoma formation and histological analysis}

Nine-week-old male NOD/SCID mice (Laboratory Animal Research Center, Bungdang CHA Medical Center, CHA University, Seongnam, Republic of Korea) were maintained in an air-conditioned animal house under specific pathogen-free conditions. To investigate teratoma formation, PD-MSCs ${ }^{\text {PRL-1 }}\left(5 \times 10^{5}\right.$ cells) were directly injected into each testis (TP; $n=$ 2). Control mice were not injected with cells (Con; $n=2$ ). After 14 weeks, the testes were collected, and all mice were sacrificed. The testes were fixed in $10 \%$ neutral buffered formalin and embedded in paraffin. Sections were stained with hematoxylin and eosin (H\&E). In all animal experimental processes, protocols were approved by the Institutional Animal Care Use Committee (IACUC) of CHA University, Seongnam, Korea (IACUC-180023).

\section{Western blotting}

Total protein was isolated lysis buffer (Sigma-Aldrich). The protein lysates were separated by 8 to $12 \%$ sodium dodecyl sulfate polyacrylamide gel electrophoresis (SDSPAGE) and transferred to polyvinylidene difluoride 
Table 2 Primer sequences using quantitative real time polymerase chain reaction

\begin{tabular}{|c|c|c|c|}
\hline Genes & & Primer sequences & $\mathrm{Tm}$ \\
\hline \multirow[t]{2}{*}{ OC } & Forward & 5'-AGTGAGAGGCAACCTGGAGA-3' & 52 \\
\hline & Reverse & 5'-GTGAAGTGAGGGCTCCCATA-3' & \\
\hline \multirow[t]{2}{*}{ COL1A1 } & Forward & 5'-TTCTTGACTGGGACCTTGTC-3' & 52 \\
\hline & Reverse & 5'-GCTTGCCTTGCTTTGAAGCA-3' & \\
\hline \multirow[t]{2}{*}{ Adipsin } & Forward & 5'-GGGCAGCGTGTACTTATCCT-3' & 52 \\
\hline & Reverse & 5'-AGAACCCCAAGATGCACAAC-3' & \\
\hline \multirow[t]{2}{*}{ PPAR- $\gamma$} & Forward & 5'-GCGGCTACTACAACCAGAGC-3' & 58 \\
\hline & Reverse & 5'-GCACATGGCACGTGTATCTC-3' & \\
\hline \multirow[t]{2}{*}{ Adiponectin } & Forward & 5'-GAGCTGACGTGGAAGATGAG-3' & 55 \\
\hline & Reverse & 5'-CTTCAAGTGCTGTCTGATTCCAATG-3' & \\
\hline \multirow[t]{2}{*}{ Leptin } & Forward & 5'-ATGCTGCAAACTGACCACGC-3' & 55 \\
\hline & Reverse & 5'-GCTTCGCTITGCCAATGCTT-3' & \\
\hline \multirow[t]{2}{*}{ LPL } & Forward & 5'-TGAGTTTGCAGAAGTTTCCA-3' & 60 \\
\hline & Reverse & 5'-CCTTTGCCTCAGCATAGTTI-3' & \\
\hline \multirow[t]{2}{*}{ FABP4 } & Forward & 5'-GCATGGCCAAACCTAACATGA-3' & 55 \\
\hline & Reverse & 5'-CCTGGCCCAGTATGAAGGAAA-3' & \\
\hline \multirow[t]{2}{*}{ IGFBP1 } & Forward & 5'-GAGCCCTGCCGAATAGAAC-3' & 60 \\
\hline & Reverse & 5'-GGATCCTCTTCCCATTCCAAG-3' & \\
\hline \multirow[t]{2}{*}{ IGFBP2 } & Forward & 5'-ACATCCCCAACTGTGACAAG-3' & 60 \\
\hline & Reverse & 5'-ATCAGCTTCCCGGTGTTG-3' & \\
\hline \multirow[t]{2}{*}{ IGFBP3 } & Forward & 5'-CAGAGCACAGATACCCAGAAC-3' & 60 \\
\hline & Reverse & 5'-AGCACATTGAGGAACTTCAGG-3' & \\
\hline \multirow[t]{2}{*}{ IGFBP4 } & Forward & 5'-CTGACAGCTTTCGAGAGTGAG-3' & 60 \\
\hline & Reverse & 5'-GCGCATTTGAGGGAAACTTC-3' & \\
\hline \multirow[t]{2}{*}{ IGFBP5 } & Forward & 5'-ACCCAGTCCAAGTITGTCG-3' & 60 \\
\hline & Reverse & 5'-TGTAGAATCCTTTGCGGTCAC-3' & \\
\hline \multirow[t]{2}{*}{ IGFBP6 } & Forward & 5'-GTCTACACCCCTAACTGCG-3' & 60 \\
\hline & Reverse & 5'-CTCTGTTGGTCTCTGCGG-3' & \\
\hline \multirow[t]{2}{*}{ |GFBP7 } & Forward & 5'-GCCCAGAAAAGCATGAAGTAAC-3' & 60 \\
\hline & Reverse & 5'-TTTATAGCTCGGCACCTTCAC-3' & \\
\hline \multirow[t]{2}{*}{ ITGA4 } & Forward & 5'-AGAGAGACAATCAGTGGTTGG-3' & 55 \\
\hline & Reverse & 5'-TCAGTTCTGTTCGTAAATCAGG-3' & \\
\hline \multirow[t]{2}{*}{ ITGB7 } & Forward & 5'-AGCAGCAACAACTCAACTGG-3' & 55 \\
\hline & Reverse & 5'-TTACAGACCCACCCTTCCTCT-3' & \\
\hline \multirow[t]{2}{*}{ FAK } & Forward & 5'-GAAGCATTGGGTCGGGAACTA-3' & 55 \\
\hline & Reverse & 5'-CTCAATGCAGTTTGGAGGTGC-3' & \\
\hline \multirow[t]{2}{*}{ GAPDH } & Forward & 5'-TCCTTCTGCATCCTGTCAGCA-3' & 58 \\
\hline & Reverse & 5'-CAGGAGATGGCCACTGCCGCA-3' & \\
\hline
\end{tabular}

(PVDF) membranes, which were then blocked in 5\% bovine serum albumin and incubated overnight at $4{ }^{\circ} \mathrm{C}$ with the following primary antibodies: anti-phosphoPI3K p110 $\alpha$ (1:1000, Cell Signaling Technology, Danvers, MA), anti-phospho-AKT (1:1000, Cell Signaling
Technology), anti-phospho-mTOR (11,000, Abcam), anti-phospho-FAK (1:1000, Cell Signaling Technology), anti-PPAR- $\gamma$ (1:500, Santa Cruz Biotechnology, Dallas, TX), anti-leptin (1:500, R\&D systems), anti-TNF- $\alpha$ (1:500, Santa Cruz Biotechnology), and anti-GAPDH (1:3000, AbFrontier, Seoul, Republic of Korea). The membranes were then incubated with horseradish peroxidase (HRP)conjugated secondary antibodies (Bio-Rad, Hercules, CA, USA), and the bands were detected using an enhancedchemiluminescence reagent (Bio-Rad).

\section{Karyotyping analysis}

Naïve and PD-MSCs ${ }^{\text {PRL-1 }}$ analyzed karyotypes, respectively using G-banding techiniques. The karyotype analysis was interpreted according to the International System for Human Cytogenomic Nomenclature (ISCN 2016) and visualized under light microscope (Axioskop2 plus, Zeiss, Germany).

\section{Human cytokine array}

Cell culture supernatants in naïve and PD-MSCs ${ }^{\text {PRL-1 }}$ were collected and analyzed according to the manufacturer's protocol using Human Proteome Profiler ${ }^{\text {Thu }}$ Cytokine Array Kit (R\&D Systems).

\section{Statistical analysis}

Data analyses were performed using GraphPrism version 5.0 (GraphPad Software, CA, USA) and statistically significant differences were assessed using twotailed unpaired Student's t-test or nonparametric statistical test by Mann-Whitney $U$ and Kruskal-Wallis test at a significance level of less than 0.05. All experiments were analyzed in duplicate or triplicate.

\section{Results}

Characterization of PD-MSCs modified with the PRL-1 gene PD-MSCs were transfected with the PRL-1 gene using a nonviral AMAXA system (Fig. 1a). After transfection, PRL-1 expression in PD-MSCs was verified by expression of the GFP reporter gene (Fig. 1b). The mRNA and protein expression levels of PRL- 1 in PD-MSCs ${ }^{\text {PRL-1 }}$ were significantly higher than those in naïve cells (Fig. 1c, d, $" p<$ $0.05)$. We analyzed the mRNA expression of genes associated with stemness markers (e.g. Oct4, Nanog and Sox2, telomerase reverse transcriptase; TERT, and HLA-G) in PD-MSCs ${ }^{\text {PRL-1 }}$. As expected, PD-MSCs ${ }^{\text {PRL-1 }}$ were well maintained at passages 1 and 6 (Fig. 1e). To identify the phenotypes of PD-MSCs, the cell surface markers on PDMSCs $^{\text {PRL-1 }}$ were analyzed by flow cytometry. PDMSCs $^{\text {PRL-1 }}$ were positive for the expression of the MSC markers CD13, CD90, and CD105 but were negative for the hematopoietic lineage markers CD34 and HLA-DR; however, the HLA class I molecule HLA-ABC was highly expressed (Fig. 1f). Additionally, no teratoma formation 


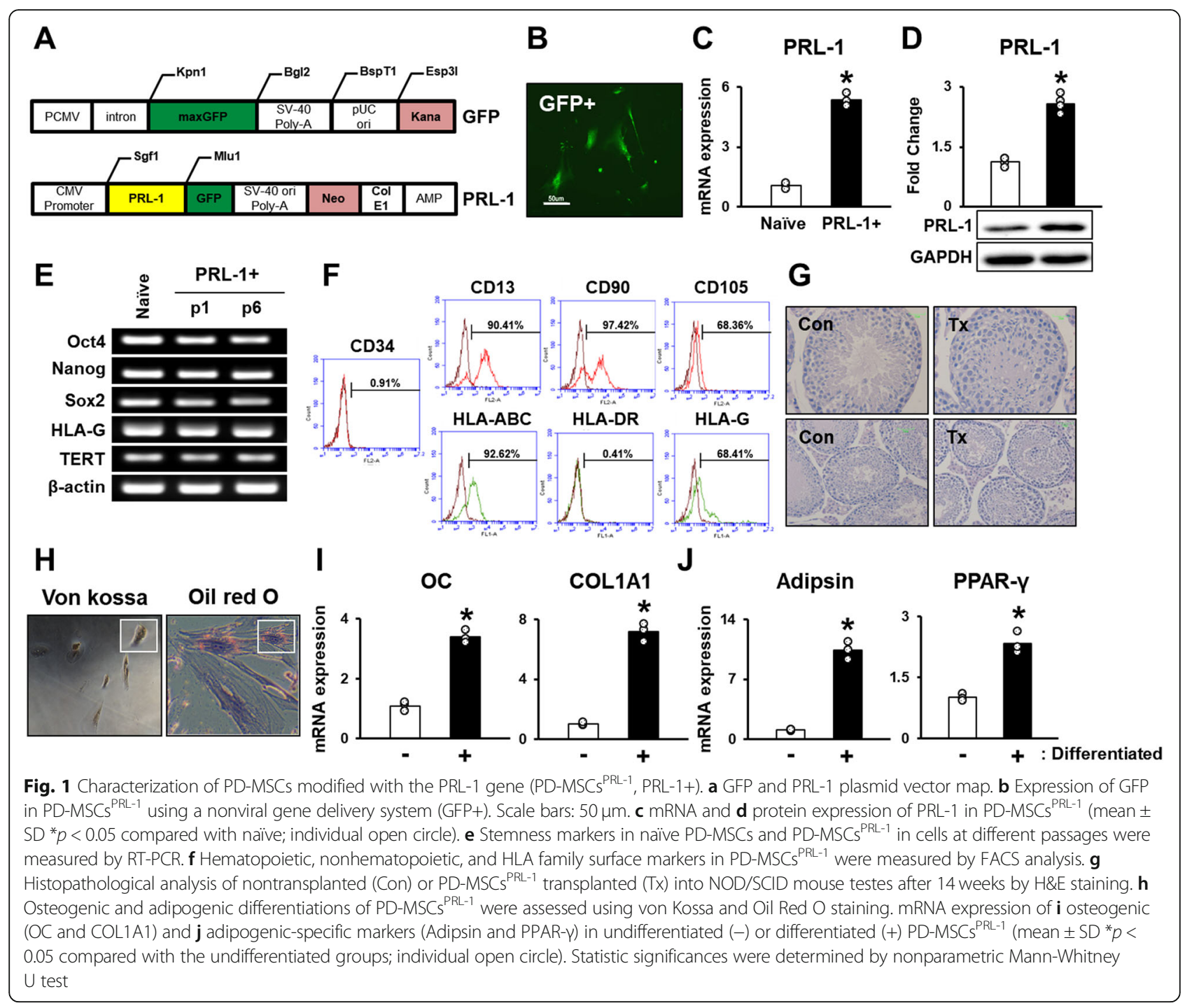

was observed after transplantation of PD-MSCs ${ }^{\text {PRL-1 }}$ (Fig. $1 \mathrm{~g})$. Karyotyping of naïve and PD-MSCs ${ }^{\text {PRL-1 }}$ was diploid and genetically stable (Supplementary Fig. 1). The differentiation into mesodermal lineage was induced in PD$\mathrm{MSCs}^{\mathrm{PRL}-1}$. Osteogenic and adipogenic differentiation of PD-MSCs ${ }^{\text {PRL-1 }}$ were evaluated by positive staining with von Kossa and Oil Red O, respectively (Fig. 1h). We previously confirmed the multidifferentiation potential of naïve MSCs [19]. Osteogenic-specific markers (Osteocalcin; OC and Collagen Type 1 alpha 1; COL1A1) and adipogenicspecific markers (Adipsin and PPAR- $\gamma$ ) were increased in differentiated PD-MSCs ${ }^{\text {PRL-1 }}$ (Fig. $1 \mathrm{i}, \mathrm{j}^{\mathrm{j},{ }^{*}} p<0.05$ ). These findings suggest that PD-MSCs ${ }^{\text {PRL-1 }}$ maintain characteristics to those of naïve cells.

PD-MSCs ${ }^{\text {PRL-1 }}$ inhibit adipogenesis in OFs from GO patients To evaluate the effects of PD-MSCs ${ }^{\text {PRL-1 }}$ on adipogenesis in OFs from GO patients, adipogenesis was induced in normal and GO-derived OFs for 4 days, followed by 6 days of maturation. After 10 days of in vitro maturation, differentiated GO-derived OFs were indirectly cocultured with naïve PD-MSCs and PD-MSCs ${ }^{\text {PRL-1 }}$ (Fig. 2a). Normal and GO-derived OFs were stained using Oil Red $\mathrm{O}$ to visualize lipid accumulation (Fig. 2b). The mRNA expression levels of adipogenic-specific markers (e.g. adipsin, adiponectin, PPAR- $\gamma$, leptin, lipoprotein lipase; LPL, and fatty acidbinding protein 4; FABP4) in OFs from GO that were cocultured with naïve PD-MSCs and PD-MSCs ${ }^{\text {PRL-1 }}$ were decreased compared to those of cells that were not cocultured. Interestingly, leptin and LPL expression in cocultured PD-MSCs ${ }^{\text {PRL-1 }}$ was significantly decreased (Fig. 2c, $" p<0.05, \# p<0.05$ ). These findings suggest that PD-MSCs ${ }^{\text {PRL-1 }}$ downregulate the gene expression of adipogenic markers and inhibit adipogenesis in OFs from GO patients. 


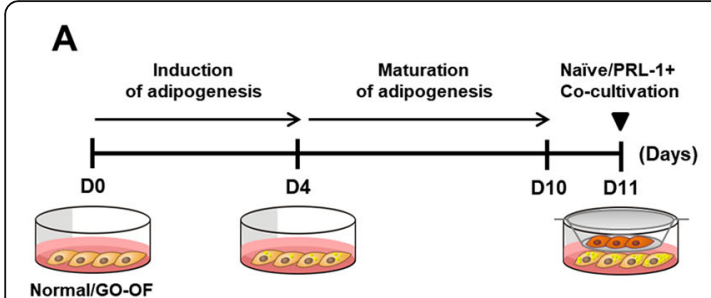

Normal/GO-OF
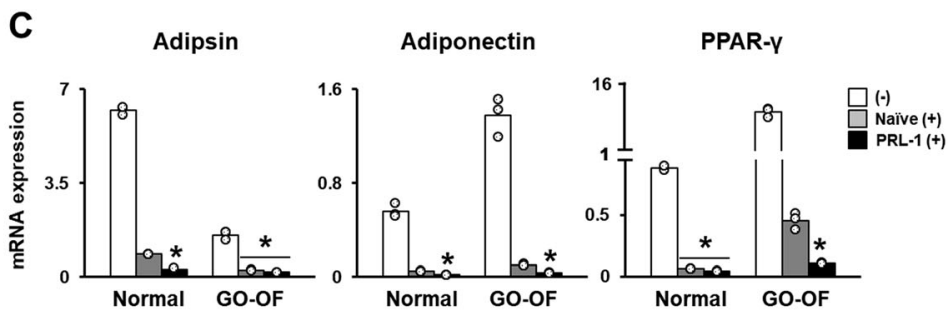

B

Co-culture
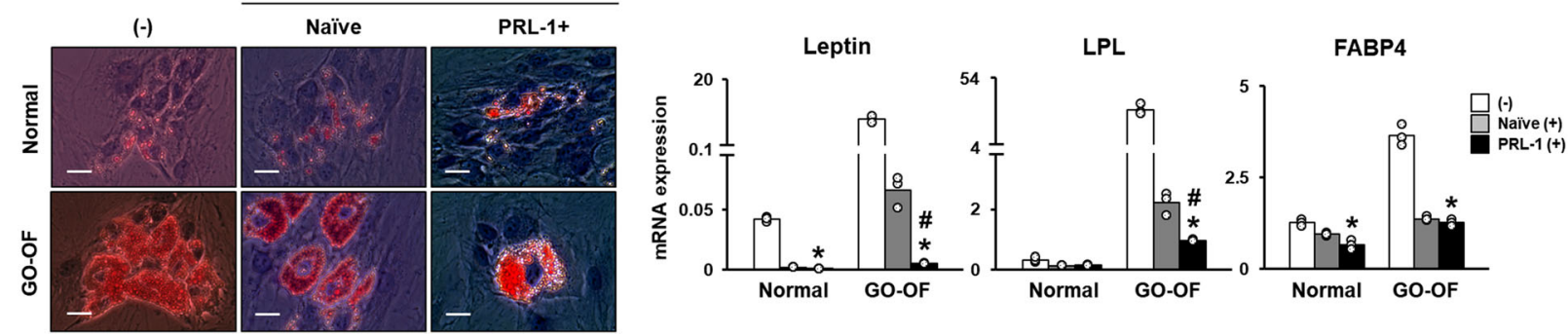

Fig. 2 PD-MSCS ${ }^{\text {PRL-1 }}$ inhibit adipogenesis in OFs from GO patients. a Schematic diagram describing naïve PD-MSCS (Naïve (+)) and PD-MSCS ${ }^{\text {PRL-1 }}$ (PRL-1 (+)) coculture with normal ( $n=3$; individual open circle) and GO-derived OFs (GO-OF; $n=3$; individual open circle) that underwent adipogenic differentiation. During the first 4 days, adipogenesis was induced in normal and GO-OFs. For 10 days, normal and GO-OFs were maintained and underwent adipogenic maturation. Naiive PD-MSCS and PD-MSCS ${ }^{\text {PLL-1 }}$ were cocultured in the transwell insert system for $24 \mathrm{~h}$. b Representative images of adipogenic differentiation of normal and GO-OFs cocultured with naive and PD-MSCS ${ }^{\text {PRL-1 }}$. Scale bars: $100 \mu \mathrm{m}$. c qRT-PCR analysis of mRNA expression of adipogenic markers in normal and GO-OFs that underwent adipogenic differentiation and were cocultured with naive PD-MSCs and PD-MSCS ${ }^{\text {PRL-1. }}$. (mean \pm SD ${ }^{*} p<0.05$ compared with the noncoculture $(-)$ groups) (mean \pm SD \#p $<0.05$ compared with the naiive coculture groups; individual open circle). Statistic significances were determined by nonparametric Kruskal-Wallis test

PD-MSCs ${ }^{\text {PRL-1 }}$ promote the expression of IGFBP genes IGFBPs control IGF-1R actions by regulating the bioavailability of the ligands IGF-1 and IGF-2 and have been shown to have inhibitory effects on adipogenesis in human visceral adipocytes. We previously analyzed whether naive $\mathrm{PD}-\mathrm{MSCs}$ and PD-MSCs ${ }^{\text {PRL-1 }}$ secreted IGFBP2 and 3 with human cytokine XL profiler arrays (Supplementary Fig. 2). Therefore, we investigated whether PD-MSCs ${ }^{\text {PRL-1 }}$ promoted the expression of IGFBPs. qRT-PCR analysis revealed that the expression levels of IGFBP-2, $-4,-6$, and -7 in PD-MSCs ${ }^{\text {PRL-1 }}$ were significantly elevated, although IGFBP-3 and -5 were reduced compared to those in naïve cells. These data show that PD-MSCs ${ }^{\text {PRL-1 }}$ upregulate the expression of IGFBP-2, $-4,-6$, and -7 and may control IGFBP secretion (Fig. $3, " p<0.05, \# p<0.05$ ).

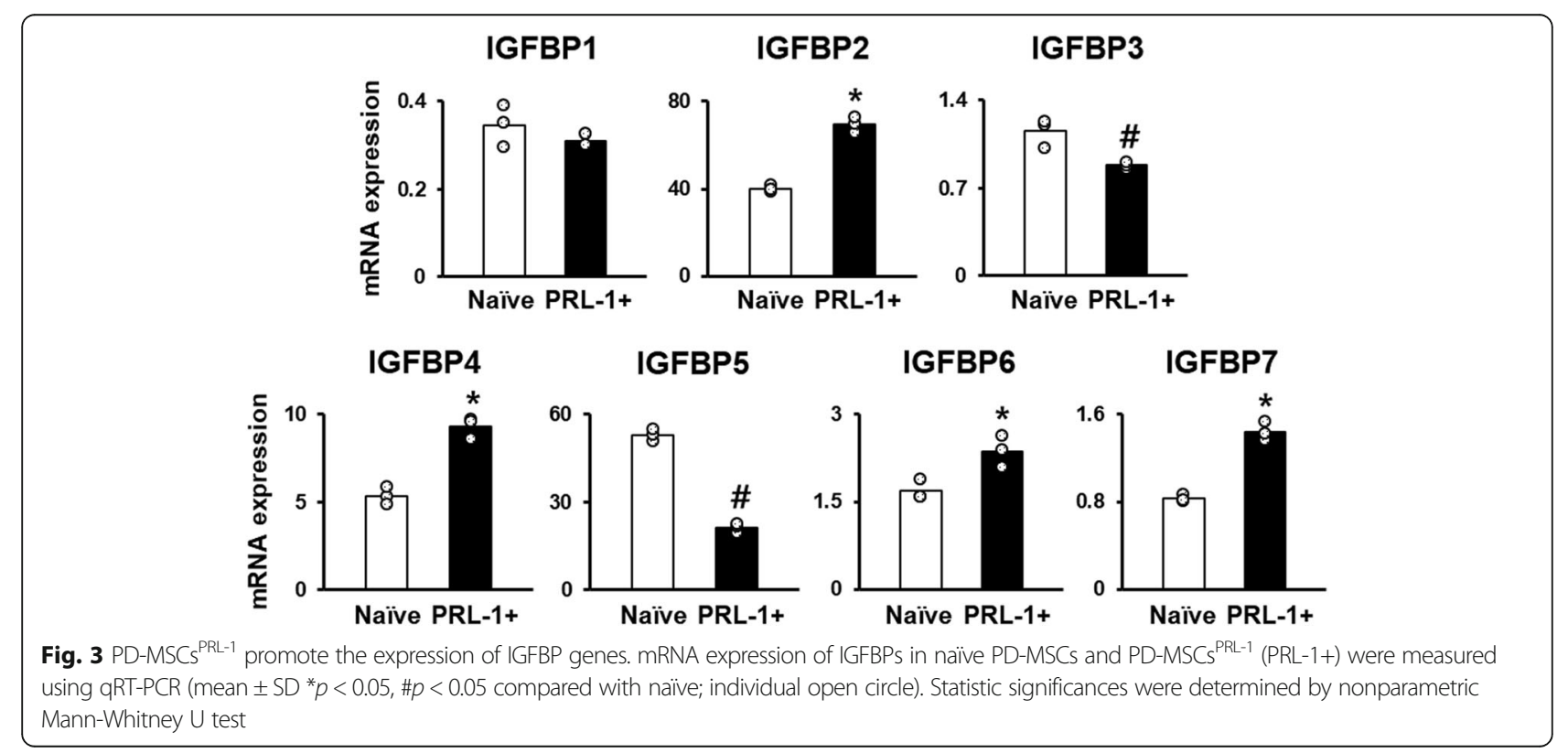


IGFBPs secreting PD-MSCs ${ }^{\text {PRL-1 }}$ inhibit adipogenesis via upregulation of FAK and downregulation of the PI3K/ AKT/mTOR signaling pathway

We confirmed that the increased expression of adipogenicspecific genes in OFs from GO patients was downregulated by PD-MSCs ${ }^{\text {PRL-1 }}$. Moreover, the protein expressions of PPAR $-\gamma$ and TNF- $\alpha$ in OFs derived from GO patients cocultured with PD-MSCs ${ }^{\text {PRL-1 }}$ were decreased compared to that of OFs that were cocultured with naïve PD-MSCs (Fig. 4a, b). Interestingly, leptin expression in the PD$\mathrm{MSCs}^{\mathrm{PRL}-1}$ coculture group was markedly decreased compared with that in the naïve coculture group (Fig. $4 \mathrm{c},{ }^{*} p<$ $0.05, \# p<0.05)$. To further investigate the mechanism by which PD-MSCs ${ }^{\text {PRL-1 }}$ inhibit IGF-1-mediated adipogenesis signaling, we analyzed the expression levels of PI3K/AKT/ mTOR pathway members by western blot analysis. The protein expression of phosphorylated PI3K, AKT, and mTOR in OFs from GO patients cocultured with PDMSCs $^{\text {PRL-1 }}$ was significantly downregulated compared to that of cells that were not cocultured.

Interestingly, PD-MSC ${ }^{\text {PRL-1 }}$ coculture with OFs also decreased the levels of phosphorylated PI3K and the expression of downstream AKT and mTOR compared with those of the naïve coculture group (Fig. 4e). In general, integrins are transmembrane receptors that facilitate cellextracellular matrix adhesion and can interact with IGFBPs. To confirm that the PD-MSC ${ }^{\mathrm{PRL}-1}$-mediated increase in IGFBPs in OFs from GO patients contributes to regulating the adipogenic effect through the integrin signaling pathway, we investigated the expression of ITGA4 and ITGB7 and the integrin downstream signaling factor FAK in normal and GO-derived OFs cocultured with PD-MSCs ${ }^{\text {PRL-1. }}$ The mRNA expression levels of ITGA4 and ITGB7 in normal and GO-derived OFs cocultured with PD-MSCs ${ }^{\text {PRL-1 }}$ were higher than those in noncocultured OFs (Fig. 4f, g, $* p<0.05, \# p<0.05)$. Moreover, the mRNA expression of FAK, which is a downstream factor of ITGA4 and ITGB7, in normal and GO-derived OFs cocultured with PDMSCs $^{\text {PRL-1 }}$ was significantly higher than that in OFs cocultured with naïve PD-MSCs (Fig. 4h, " $p<0.05$, \#p<0.05). As shown in Fig. 4e, the mRNA level of FAK was consistent with the protein level. These findings suggest that enhanced IGFBP expression by PD-MSCs ${ }^{\text {PRL-1 }}$ promotes ITGA4 and ITGB7 signaling, which leads to FAK activation and downregulates the $\mathrm{PI} 3 \mathrm{~K} / \mathrm{AKT} / \mathrm{mTOR}$ signaling pathway, resulting in inhibition of OF adipogenesis (Fig. 5).

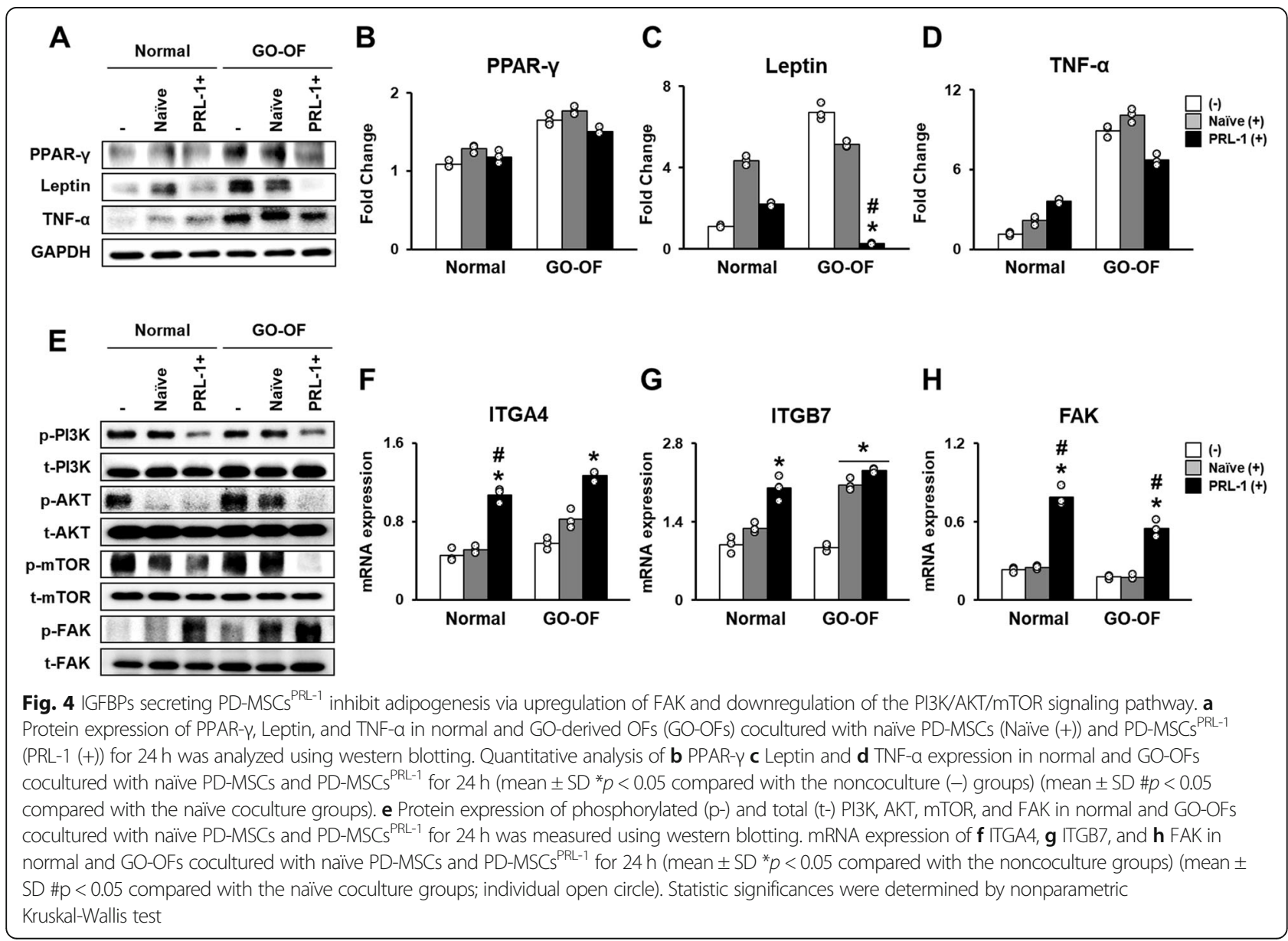




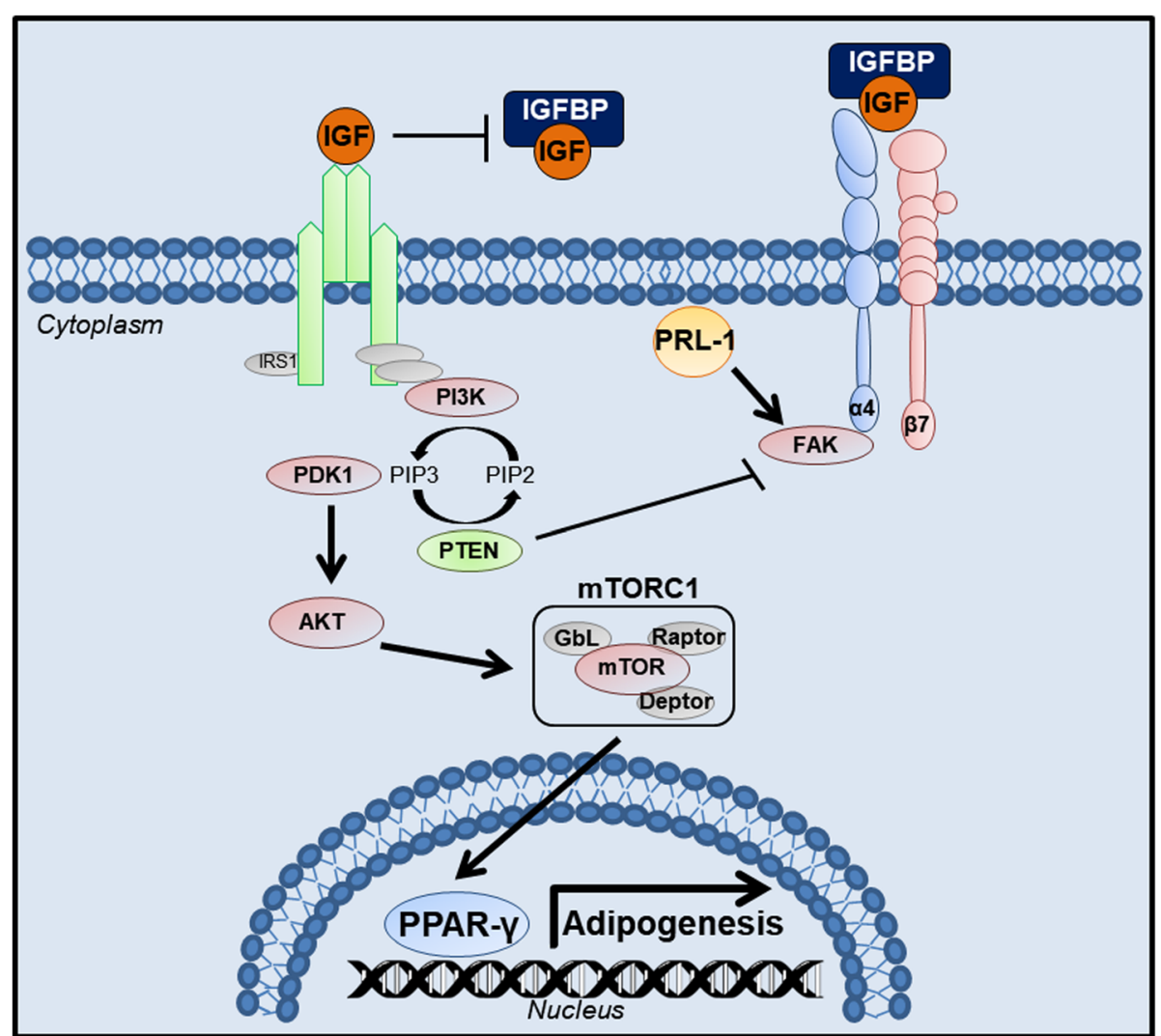

Fig. 5 Summarized diagram proposing that IGFBPs secreting PD-MSCS ${ }^{\text {PRL-1 }}$ are the key antiadipogenic factors inhibiting adipogenesis in OFs from $\mathrm{GO}$ patients through upregulation of FAK and downregulation of the PI3K/AKT/mTOR signaling pathway

\section{Discussion}

MSCs have immunomodulatory roles in autoimmune diseases, including GO [20]. Because medical therapies, including corticosteroids and radiotherapy, for patients with GO lead to side effects and the development of malignancies, understanding the molecular mechanisms of de novo adipogenesis and the main symptoms of GO is critical in developing therapeutic applications. In particular, MSCs have anti-inflammatory and immunosuppressive effects on antigen presenting cells and secrete soluble factors, including adipokines, exosomes, and miRNAs [21-24]. Previous reports showed that PDMSCs have more immunological advantages than other MSCs, as evidenced by the increased expression of HLA-G and the cytokines of IL-2, IL-4, IL-13, and GMCSF [12]. However, MSC aging results in limited selfrenewal abilities and age-associated decreases in cellular numbers and functions [25]. Therefore, gene modification using gene delivery systems overcomes the limited functions of MSCs to provide effective therapeutic results [26]. A recent report revealed that genetically modified MSCs overexpressing IL-35 could be applied in autoimmune diseases to overcome the complications of long-term immunosuppression [27]. Especially, AMAXA technique provides critical opportunities for hard-totransfect primary cell line including MSCs. In our previous study, we generated TERT-overexpressing PD-MSCs using a nonviral AMAXA system to study the underlying regulatory mechanisms of self-renewal [28]. In addition, PD-MSC ${ }^{\text {PRL-1 }}$ transplantation in hepatic failure model indicated therapeutic effects including anti-fibrotic and proliferative potentials compared to naïve PD-MSCs [29].

PRL-1 is a member of a subgroup of related protein tyrosine phosphatases contacting a C-terminal prenylation motif [14]. C-terminal residues and cellular redox environments are controlled by the enzymatic activity of PRL-1 [30]. In oxidative-stressed retinas and photoreceptors, modulation of PRL-1 activity regulates redox conditions [16]. We hypothesized that PD-MSCs ${ }^{\text {PRL-1 }}$ would regulate oxidative conditions and reduce adipogenesis in OFs from GO patients.

Because little is known about the efficacy of PDMSC $^{\text {PRL-1 }}$-mediated inhibition of adipogenesis in OFs from $\mathrm{GO}$ patients, we further analyzed the functional enhancement of PRL-1 in PD-MSCs generated using a nonviral AMAXA system. OFs isolated from patients with GO are capable of adipocyte differentiation [3]. In orbital adipose tissues and in vitro GO-derived OFs after 
differentiation, enhanced adiponectin, leptin and PPAR$\gamma$ were positively correlated [31]. Previous reports demonstrated that IGF-1 expression was enhanced and PI3K was activated by upregulating PPAR- $\gamma$ in the orbital fatty connective tissue of patients with GO [32]. In general, IGF binds to IGFBPs. Individual IGFBPs act to increase or attenuate the IGF signaling pathway [33]. IGF-1R/ mTOR is associated with differentiation of adiposederived stem cells (ASCs) [34] and IGFBPs by ASCs regulate the IGF1 effect [35].

Especially, IGFBP2 prevents adipogenesis [36], and IGFBP3 interferes with PPAR- $\gamma$-dependent processes to impair adipocyte differentiation [37]. Overexpressed IGFBP2 inhibits both lipogenesis and adipogenesis in visceral adipocytes, and this process involves cell surface association of IGFBP2 and activation of integrin signaling pathway [38]. Similarly, IGFBP4 controls the expression of insulin and IGF1 in mouse adipose tissue expansion [39]. We previously analyzed whether naïve PD-MSCs and PDMSCs $^{\text {PRL-1 }}$ significantly secreted IGFBPs using a cytokine array (Supplementary Fig. 2). Based on the results, we found that PD-MSCs ${ }^{\text {PRL-1 }}$ secreted IGFBP2, $-4,-6$, and 7 and inhibited adipogenesis. Previously, we confirmed that naïve PD-MSCs have anti-adipogenic effects in the GO animal model [17]. However, our studies are needed to further investigate the molecular mechanism of PD-MSC ${ }^{\text {PRL-1 }}$ transplantation in animals undergoing experimental GO. In the present study, these findings suggest that IGFBPs secreting PD-MSCs ${ }^{\text {PRL-1 }}$ through ITGA4- and ITGB7 decreased PPAR- $\gamma$-dependent processes via downregulation of PI3K/AKT/mTOR activities and inhibited adipogenesis.

\section{Conclusions}

In this study, we showed that PD-MSCs modified with the PRL-1 gene using nonviral transfection method efficiently overexpressed the PRL-1 protein and maintained the phenotype and multilineage differentiation properties of MSCs. PD-MSCs ${ }^{\text {PRL-1 }}$ induced IGFBP expression and inhibited adipogenesis via upregulation of FAK and downregulation of the PI3K/AKT/mTOR signaling pathway in OFs from GO patients. In this study, we focused on overcoming the medical problems of GO patients, and functional enhancement of PD-MSCs by nonviral gene modification provides novel insight into nextgeneration MSC-based cell therapy for future clinical trials in immunological diseases.

\section{Supplementary Information}

The online version contains supplementary material available at https://doi. org/10.1186/s13287-020-01982-3.

Additional file 1: Supplementary Fig. 1 Karyotyping in naïve PDMSCs (Naïve) and PD-MSCs ${ }^{\text {PRL-1 }}$ (PRL-1+).
Additional file 2: Supplementary Fig. 2 secreted IGFBP2 and 3 in naïve PD-MSCs (Naïve) and PD-MSCs ${ }^{\text {PRL-1 }}$ (PRL-1+) culture supernatants by cytokine array. Each protein is represented by duplicate spots on the respective membrane (mean \pm SD ${ }^{*} p<0.05$ compared with naïve).

\section{Abbreviations}

ASC: adipose-derived stem cells; AMPK: AMP-activated protein kinase: COL1A1: Collagen type 1 alpha 1; FABP4: Fatty acid-binding protein 4; GO: Graves' ophthalmopathy; HLA: Human leukocyte antigen; HMG-COA: $\beta$ Hydroxy $\beta$-methylglutaryl-coenzyme A; IGFBPs: Insulin-like growth factorbinding proteins; IGF-1R: Insulin-like growth factor 1 receptor; ITGA4: Integrin alpha 4; LPL: Lipoprotein lipase; OC: Osteocalcin; OF: Orbital fibroblast; PDMSCs: Placenta-derived mesenchymal stem cells; PPAR- $\gamma$ : Peroxisome proliferator-activated receptor-gamma; PRL-1: Phosphatase of regenerating liver-1; TERT: Telomerase reverse transcriptase; TSHR: Thyroid-stimulating hormone receptor

\section{Acknowledgements}

Not applicable.

\section{Authors' contributions}

JYK contributed to the analysis and interpretation of data and manuscript writing. $\mathrm{H}-\mathrm{JL}$ did the data analysis. HL provided OF. SP and GJK reviewed the manuscript. GJK did financial support, and final approval of manuscript. All authors read and approved the final manuscript.

\section{Funding}

This research was supported by grants of the Korea Health Technology R\&D Project through the Korea Health Industry Development Institute (KHIDI), funded by the Ministry of Health \& Welfare, Republic of Korea (grant number: HI16C1599) and by Basic Science Research Program through the National Research Foundation of Korea (NRF) funded by the Ministry of Education (grant number: 2019R1/1A1A01057255).

Availability of data and materials

All data and materials are available upon request.

\section{Ethics approval and consent to participate}

The process of obtaining orbital adipose tissues was approved by the Institutional Review Board of Bundang CHA Medical Center, Seongnam, Republic of Korea (IRB-2018-01-007), and placenta tissues were collected for research purposes by the Institutional Review Board of CHA Gangnam Medical Center, Seoul, Republic of Korea (IRB 07-18). All patients consented to the proper use for research. In all animal experimental processes, protocols were approved by the Institutional Animal Care Use Committee (IACUC) of CHA University, Seongnam, Korea (IACUC-180023). All patients provided written informed consent.

\section{Consent for publication}

Not applicable.

\section{Competing interests}

The authors declare that they have no competing interests.

\section{Author details}

'Department of Biomedical Science, CHA University, Seongnam 13488, Republic of Korea. ${ }^{2}$ Center for Non-Clinical Development, CHA Advanced Research Institute CHA University, Seongnam 13488, Republic of Korea.

${ }^{3}$ Department of Ophthalmology, CHA Bundang Medical Center CHA

University, Seongnam 13496, Republic of Korea.

Received: 17 February 2020 Accepted: 20 October 2020

Published online: 05 November 2020

References

1. Bahn RS. Graves' ophthalmopathy. N Engl J Med. 2010;362(8):726-38.

2. Mohyi M, Smith TJ. IGF1 receptor and thyroid-associated ophthalmopathy. J Mol Endocrinol. 2018;61(1):T29-43.

3. Mimura LY, Villares SM, Monteiro ML, Guazzelli IC, Bloise W. Peroxisome proliferator-activated receptor-gamma gene expression in orbital adipose/ 
connective tissues is increased during the active stage of Graves' ophthalmopathy. Thyroid. 2003;13(9):845-50.

4. Li B, Smith TJ. PI3K/AKT pathway mediates induction of IL-1RA by TSH in fibrocytes: modulation by PTEN. J Clin Endocrinol Metab. 2014;99(9):3363-72.

5. Ponto KA, Zang S, Kahaly GJ. The tale of radioiodine and Graves' orbitopathy. Thyroid. 2010:20(7):785-93.

6. Shams PN, Ma R, Pickles T, Rootman J, Dolman PJ. Reduced risk of compressive optic neuropathy using orbital radiotherapy in patients with active thyroid eye disease. Am J Ophthalmol. 2014;157(6):1299-305.

7. Giotaki Z, Fountas A, Tsirouki T, Bargiota A, Tigas S, Tsatsoulis A. Adrenal reserve following treatment of Graves' orbitopathy with intravenous glucocorticoids. Thyroid. 2015;25(4):462-3.

8. Moleti M, Giuffrida G, Sturniolo G, Squadrito G, Campenni A, Morelli S, et al. Acute liver damage following intravenous glucocorticoid treatment for Graves' ophthalmopathy. Endocrine. 2016;54(1):259-68.

9. Mazonakis M, Tzedakis A, Lyraraki E, Damilakis J. Risk of developing radiogenic cancer following photon-beam radiotherapy for Graves' orbitopathy. Med Phys. 2018;45(10):4775-82.

10. Diehl R, Ferrara F, Muller C, Dreyer AY, McLeod DD, Fricke S, Boltze J. Immunosuppression for in vivo research: state-of-the-art protocols and experimental approaches. Cell Mol Immunol. 2017;14(2):146-79.

11. Cervelli V, Bocchini I, Di Pasquali C, De Angelis B, Cervelli G, Curcio CB, et al. P.R.L. platelet rich lipotransfert: our experience and current state of art in the combined use of fat and PRP. Biomed Res Int. 2013;2013:434191.

12. Lee JM, Jung J, Lee HJ, Jeong SJ, Cho KJ, Hwang SG, Kim GJ. Comparison of immunomodulatory effects of placenta mesenchymal stem cells with bone marrow and adipose mesenchymal stem cells. Int Immunopharmacol. 2012; 13(2):219-24.

13. Rios P, Li X, Kohn M. Molecular mechanisms of the PRL phosphatases. FEBS J. 2013:280(2):505-24.

14. Si X, Zeng Q, Ng CH, Hong W, Pallen CJ. Interaction of farnesylated PRL-2, a protein-tyrosine phosphatase, with the beta-subunit of geranylgeranyltransferase II. J Biol Chem. 2001;276(35):32875-82.

15. Gao J, Liao J, Yang GY. CAAX-box protein, prenylation process and carcinogenesis. Am J Transl Res. 2009;1(3):312-25.

16. Yu L, Kelly U, Ebright JN, Malek G, Saloupis P, Rickman DW, McKay BS, Arshavsky $\mathrm{V}$, Bowes RC. Oxidative stress-induced expression and modulation of phosphatase of regenerating Liver-1 (PRL-1) in mammalian retina. Biochim Biophys Acta. 2007;1773(9):1473-82.

17. Park M, Banga JP, Kim GJ, Kim M, Lew H. Human placenta-derived mesenchymal stem cells ameliorate orbital adipogenesis in female mice models of Graves' ophthalmopathy. Stem Cell Res Ther. 2019;10(1):246.

18. Lee MJ, Jung J, Na KH, Moon JS, Lee HJ, Kim JH, et al. Anti-fibrotic effect of chorionic plate-derived mesenchymal stem cells isolated from human placenta in a rat model of CCl(4)-injured liver: potential application to the treatment of hepatic diseases. J Cell Biochem. 2010;111(6):1453-63.

19. Seok J, Jung HS, Park S, Lee JO, Kim CJ, Kim GJ. Alteration of fatty acid oxidation by increased CPT1A on replicative senescence of placenta-derived mesenchymal stem cells. Stem Cell Res Ther 2020;11(1):1.

20. De Miguel MP, Fuentes-Julian S, Blazquez-Martinez A, Pascual CY, Aller MA, Arias J, Arnalich-Montiel F. Immunosuppressive properties of mesenchymal stem cells: advances and applications. Curr Mol Med. 2012;12(5):574-91.

21. Gentile P, Garcovich S. Concise Review: Adipose-Derived Stem Cells (ASCs) and Adipocyte-Secreted Exosomal microRNA (A-SE-miR) Modulate Cancer Growth and proMote Wound Repair. J Clin Med. 2019;8(6):855.

22. Gentile P, Piccinno MS, Calabrese C. Characteristics and Potentiality of Human Adipose-Derived Stem Cells (hASCs) Obtained from Enzymatic Digestion of Fat Graft. Cells. 2019;8(3):282.

23. Gentile P, Calabrese C, De Angelis B, Pizzicannella J, Kothari A, Garcovich S. Impact of the Different Preparation Methods to Obtain Human AdiposeDerived Stromal Vascular Fraction Cells (AD-SVFs) and Human AdiposeDerived Mesenchymal Stem Cells (AD-MSCs): Enzymatic Digestion Versus Mechanical Centrifugation. Int J Mol Sci. 2019;20(21):5471.

24. Scioli MG, Storti G, D'Amico F, Gentile P, Kim BS, Cervelli V, Orlandi A. Adipose-Derived Stem Cells in Cancer Progression: New Perspectives and Opportunities. Int J Mol Sci. 2019;20(13):3296.

25. Li Y, Wu Q, Wang Y, Li L, Bu H, Bao J. Senescence of mesenchymal stem cells (review). Int J Mol Med. 2017;39(4):775-82.

26. Nowakowski A, Walczak P, Lukomska B, Janowski M. Genetic engineering of Mesenchymal stem cells to induce their migration and survival. Stem Cells Int. 2016;2016:4956063.
27. Guo H, Li B, Wang W, Zhao N, Gao H. Mesenchymal stem cells overexpressing IL-35: a novel immunosuppressive strategy and therapeutic target for inducing transplant tolerance. Stem Cell Res Ther. 2018;9(1):254.

28. Lee HJ, Choi JH, Jung J, Kim JK, Lee SS, Kim GJ. Changes in PTTG1 by human TERT gene expression modulate the self-renewal of placentaderived mesenchymal stem cells. Cell Tissue Res. 2014;357(1):145-57.

29. Kim JY, Jun JH, Park SY, Yang SW, Bae SH, Kim GJ. Dynamic Regulation of miRNA Expression by Functionally Enhanced Placental Mesenchymal Stem Cells Promotes Hepatic Regeneration in a Rat Model with Bile Duct Ligation. Int J Mol Sci. 2019;20(21):5299.

30. Skinner AL, Vartia AA, Williams TD, Laurence JS. Enzyme activity of phosphatase of regenerating liver is controlled by the redox environment and its C-terminal residues. Biochemistry. 2009;48(20):4262-72.

31. Kumar S, Leontovich A, Coenen MJ, Bahn RS. Gene expression profiling of orbital adipose tissue from patients with Graves' ophthalmopathy: a potential role for secreted frizzled-related protein-1 in orbital adipogenesis. J Clin Endocrinol Metab. 2005;90(8):4730-5.

32. Zhao P, Deng Y, Gu P, Wang Y, Zhou H, Hu Y, Chen P, Fan X. Insulin-like growth factor 1 promotes the proliferation and adipogenesis of orbital adipose-derived stromal cells in thyroid-associated ophthalmopathy. Exp Eye Res. 2013;107:65-73.

33. Hwa $V, O h Y$, Rosenfeld $R G$. The insulin-like growth factor-binding protein (IGFBP) superfamily. Endocr Rev. 1999;20(6):761-87.

34. Scioli MG, Bielli A, Gentile P, Cervelli V, Orlandi A. Combined treatment with platelet-rich plasma and insulin favours chondrogenic and osteogenic differentiation of human adipose-derived stem cells in three-dimensional collagen scaffolds. J Tissue Eng Regen Med. 2017;11(8):2398-410.

35. Gentile P, Garcovich S. Advances in Regenerative Stem Cell Therapy in Androgenic Alopecia and Hair Loss: Wnt pathway, Growth-Factor, and Mesenchymal Stem Cell Signaling Impact Analysis on Cell Growth and Hair Follicle Development. Cells. 2019;8(5):466.

36. Wheatcroft SB, Kearney MT, Shah AM, Ezzat VA, Miell JR, Modo M, et al. IGFbinding protein-2 protects against the development of obesity and insulin resistance. Diabetes. 2007;56(2):285-94.

37. Chan SS, Schedlich LJ, Twigg SM, Baxter RC. Inhibition of adipocyte differentiation by insulin-like growth factor-binding protein-3. Am J Physiol Endocrinol Metab. 2009;296(4):E654-63.

38. Yau SW, Russo VC, Clarke IJ, Dunshea FR, Werther GA, Sabin MA. IGFBP-2 inhibits adipogenesis and lipogenesis in human visceral, but not subcutaneous, adipocytes. Int J Obes. 2015;39(5):770-81.

39. Gealekman O, Gurav K, Chouinard M, Straubhaar J, Thompson M, Malkani S, Hartigan C, Corvera S. Control of adipose tissue expandability in response to high fat diet by the insulin-like growth factor-binding protein-4. J Biol Chem. 2014;289(26):18327-38.

\section{Publisher's Note}

Springer Nature remains neutral with regard to jurisdictional claims in published maps and institutional affiliations.

\section{Ready to submit your research? Choose BMC and benefit from:}

- fast, convenient online submission

- thorough peer review by experienced researchers in your field

- rapid publication on acceptance

- support for research data, including large and complex data types

- gold Open Access which fosters wider collaboration and increased citations

- maximum visibility for your research: over $100 \mathrm{M}$ website views per year

At BMC, research is always in progress.

Learn more biomedcentral.com/submissions 